\title{
Development of a Large-Scale Functional Brain Network during Human Non-Rapid Eye Movement Sleep
}

\author{
Victor I. Spoormaker, ${ }^{1 \star}$ Manuel S. Schröter, ${ }^{1 \star}$ Pablo M. Gleiser, ${ }^{2}$ Katia C. Andrade, ${ }^{1}$ Martin Dresler, ${ }^{1}$ Renate Wehrle, ${ }^{1}$ \\ Philipp G. Sämann, ${ }^{1}$ and Michael Czisch ${ }^{1}$ \\ ${ }^{1}$ Max Planck Institute of Psychiatry, 80804 Munich, Germany, and ${ }^{2}$ Centro Atómico Bariloche, Instituto Balseiro, Consejo Nacional de Investigaciones \\ Científicas y Técnicas, Bariloche, 8400 Río Negro, Argentina
}

Graph theoretical analysis of functional magnetic resonance imaging (fMRI) time series has revealed a small-world organization of slow-frequency blood oxygen level-dependent (BOLD) signal fluctuations during wakeful resting. In this study, we used graph theoretical measures to explore how physiological changes during sleep are reflected in functional connectivity and small-world network properties of a large-scale, low-frequency functional brain network. Twenty-five young and healthy participants fell asleep during a 26.7 min fMRI scan with simultaneous polysomnography. A maximum overlap discrete wavelet transformation was applied to fMRI time series extracted from 90 cortical and subcortical regions in normalized space after residualization of the raw signal against unspecific sources of signal fluctuations; functional connectivity analysis focused on the slow-frequency BOLD signal fluctuations between $0.03 \mathrm{and} 0.06 \mathrm{~Hz}$. We observed that in the transition from wakefulness to light sleep, thalamocortical connectivity was sharply reduced, whereas corticocortical connectivity increased; corticocortical connectivity subsequently broke down in slow-wave sleep. Local clustering values were closest to random values in light sleep, whereas slow-wave sleep was characterized by the highest clustering ratio (gamma). Our findings support the hypothesis that changes in consciousness in the descent to sleep are subserved by reduced thalamocortical connectivity at sleep onset and a breakdown of general connectivity in slow-wave sleep, with both processes limiting the capacity of the brain to integrate information across functional modules.

\section{Introduction}

Graph theoretical analysis of functional magnetic resonance imaging (fMRI) time series has revealed a small-world organization of human functional brain networks (Achard et al., 2006), with high local clustering and short path length (Bullmore and Sporns, 2009). A small-world topology is a promising model for largescale brain networks (Bassett and Bullmore, 2006) as it supports both specialized processing in local clusters and integrated processing over the entire network (Sporns et al., 2004). Small-world properties of large-scale brain networks have helped characterize aging processes (Meunier et al., 2009; Supekar et al., 2009), intelligence (van den Heuvel et al., 2009), psychiatric disorders like schizophrenia (Bassett et al., 2008), and neurodegenerative disorders (Stam, 2009; Stam et al., 2009).

Here, we apply graph theoretical analysis to study functional connectivity in sleep, as the loss of consciousness in sleep is paradoxically accompanied by similar or even increased functional connectivity (Ferri et al., 2007, 2008; Horovitz et al., 2008; Dimitriadis et al., 2009; Larson-Prior et al., 2009). Graph theory analysis of scalp electro-

Received April 20, 2010; revised July 6, 2010; accepted July 14, 2010.

This study was supported by the Max Planck Society. K.A. acknowledges personal support from the Capes Foundation, Ministry of Education, Brazil. We are grateful to Andrea Stiegler, Henning Peters, and Caroline Tully for the screening of participants; Rosa Schirmer for data management; and Armin Mann for technical support.

*V.I.S. and M.S.S. contributed equally to this work.

Correspondence should be addressed to Dr. Victor I. Spoormaker, Max Planck Institute of Psychiatry, Kraepelinstrasse 2-10,80804 Munich, Germany. E-mail: spoormaker@mpipsykl.mpg.de.

DOI:10.1523/JNEUROSCI.2015-10.2010

Copyright $\odot 2010$ the authors $\quad 0270-6474 / 10 / 3011379-09 \$ 15.00 / 0$ encephalography (EEG) during sleep revealed not only increased neocortical connectivity but also increased small-world properties on specific frequency bands (Ferri et al., 2007, 2008; Dimitriadis et al., 2009). fMRI studies on slow $(<0.1 \mathrm{~Hz})$ spontaneous blood oxygen level-dependent (BOLD) signal fluctuations during sleep have focused on so-called resting networks, and reported no or minimal changes in the default mode network during light sleep (Horovitz et al., 2008) or a trend for increased connectivity in other resting networks (Larson-Prior et al., 2009). A breakdown of the coupling between the anterior and posterior nodes of the default mode network was found to occur in slow-wave sleep (Horovitz et al., 2009; Sämann et al., 2009).

According to an information integration account of consciousness (Tononi, 2004), not the connectivity of the system per se but its capacity to integrate information across modules is critical in understanding why consciousness fades in early sleep (Tononi and Massimini, 2008). Increased levels of functional connectivity could result in lower values for information integration of the network as a whole (Tononi and Sporns, 2003), and fading consciousness as a consequence, depending on other network properties. The trend that the fading of consciousness in early non-rapid eye movement (NREM) sleep is accompanied by increased cortical connectivity would be in line with an information integration account of consciousness if a crucial node, such as the thalamus (Steriade et al., 1993; McCormick and Bal, 1997), is excluded from the network. The thalamus shows decreased activity at sleep onset (Kaufmann et al., 2006), and this temporally precedes deactivation of the cortex (Magnin et al., 2010); 
but, decreased activity could still result in high functional connectivity with cortical regions when intrinsic fluctuations remain highly synchronized.

With simultaneous EEG/fMRI measurements during all NREM stages, we aimed to examine whether light sleep is associated with increased connectivity and exclusion of the thalamus from the large-scale brain network. In slow-wave sleep, we expected a breakdown in (cortical) connectivity as demonstrated by Massimini et al. (2005) in a high-density EEG study.

\section{Materials and Methods \\ Participants}

Twenty-five young and healthy subjects participated in the study: 12 females with a mean \pm SD age of $24.8 \pm 2.5$ years; and 13 males with a mean \pm SD age of $24.7 \pm 3.2$ years. Subjects were instructed to follow a regular sleep-wake schedule with bedtimes between 11 P.M. and 8:00 A.M. in the week before the experiment, which was documented by sleep diaries. Subjects were asked to get up $\sim 3 \mathrm{~h}$ earlier on the experimental day to increase the probability of falling asleep in the MRI scanner. Wrist actigraphy was used during the night and day before the experiment to control subjects' adherence to the sleep restriction. Simultaneous EEG/ fMRI measurements started at 9:00 P.M. with EEG montage; in the MRI scanner, subjects were informed that no active participation was required in the following 2-3 h, and that they could fall asleep. The study protocol was in accordance with the Declaration of Helsinki and was approved by a local ethics review board. Participants provided their written informed consent after the procedure had been fully explained and were reimbursed for their participation.

\section{EEG/fMRI acquisition}

Polysomnographic recordings were performed using an MR-compatible EEG system placed according to the international 10/20 electrode system with $19 \mathrm{EEG}$ channels referenced against $\mathrm{FCz}$, an electrooculogram, submental electromyogram, and an electrocardiogram (sampling rate, 5 kHz; EasyCAP modified for sleep, Brain Products; VisionRecorder Version 1.03, Brain Products). fMRI was performed at 1.5 tesla (Signa LX, GE Healthcare) using an eight-channel head coil. Due to technical restrictions, a total of 800 functional whole-brain images [echo planar imaging (EPI)] (repetition time, $2000 \mathrm{~ms}$; echo time, $40 \mathrm{~ms}$; flip angle, $90^{\circ}$; $64 \times 64$ matrix, in-plane resolution $3.4 \times 3.4 \mathrm{~mm}^{2}$; slices, 25 ; slice thickness, $3 \mathrm{~mm}$; gap, $1 \mathrm{~mm}$; oriented along the anterior commissuralposterior commissural axis) were acquired per fMRI run over $26.7 \mathrm{~min}$. Sleep was evaluated online using Vision RecView software (Brain Products). This online evaluation of the polysomnographic recordings was used, in addition to the participant's subjective report, to verify whether sleep occurred or the experiment was to be repeated, resulting in a total of 40 fMRI runs.

\section{EEG analysis}

EEG data were first corrected for gradient-induced and cardioballistic artifacts (VisionAnalyzer 1.05, Brain Products). Bandwidth was set to 0.5-30 Hz. Sleep recordings were scored according to the criteria of Rechtschaffen and Kales (1968) in 20 s epochs. The hypnograms of the 40 recorded $26.7 \mathrm{~min}$ sessions were then screened for intervals consisting of 5 min of one specific, uninterrupted sleep stage with a presence of $>85 \%$ throughout the epoch, without arousals or movement artifacts in either EEG or fMRI. This resulted in 93 epochs of the following vigilance stages: wakefulness (S0: 27); sleep stage 1 (S1: 24); sleep stage 2 (S2: 24); and slow-wave sleep (SW: 18). One S1 epoch was lost due to technical problems, resulting in 92 epochs for the final analysis. Details of sleep duration, subjects' sleep architecture, and extracted epochs are displayed in Table 1 and supplemental Table S1 (available at www.jneurosci.org as supplemental material).

\section{fMRI analysis}

Preprocessing. The fMRI preprocessing steps were performed using SPM5 software (www.fil.ion.ucl.ac.uk/spm) and consisted of the following: (1) slice time correction to account for interleaved slice acquisition; (2) realignment of time series to the first image using rigid body transforma-
Table 1. Values of sleep stage recordings per fMRI run

\begin{tabular}{lc}
\hline Sleep stage & Length (min) $^{a}$ \\
\hline Wakefulness & $6.8 \pm 5.2$ \\
Sleep stage 1 & $5.9 \pm 4.2$ \\
Sleep stage 2 & $10.0 \pm 5.4$ \\
Slow-wave sleep & $4.8 \pm 5.8$ \\
Latency waking to sleep stage 2 & $6.7 \pm 4.9$ \\
\hline
\end{tabular}

${ }^{a}$ Values are mean \pm SD.

tion to correct for head motion (only head movements $<2 \mathrm{~mm}$ between two subsequent slices were accepted); and (3) linear and nonlinear spatial normalization of the images to a standard EPI template in Montreal Neurological Institute (MNI) space (SPM5 distribution) and resampling to a voxel resolution of $2 \times 2 \times 2 \mathrm{~mm}^{3}$ using a fifth degree spline interpolation.

Removal of nuisance signals. To remove unspecific signal variation from the time series, the following regressors were defined in a multiple regression model and regressed out from the spatially unsmoothed images: $1-6$, six parameters derived from the realignment step; $7-12$, their respective first-order derivatives; $13-14$, global signals within white matter and CSF; and 15-16, their respective first-order derivatives. This is in line with previous studies on thalamocortical functional connectivity (Zhang et al., 2008), which omitted the global signal from the multiple regression models as it was observed to correlate strongly with the thalamus. The resultant residual images contained signal fluctuations not explained by regressors $1-16$, and after addition of the constant value these images were used for extraction of region-based time courses.

Time course extraction and transformation. Functional images were parcellated into 90 cortical and subcortical regions using the anatomical template image created by Tzourio-Mazoyer et al. (2002). Regional time series were extracted using the mean signal of all voxels inside a particular region with the Marsbar toolbox (version 0.41) (Brett et al., 2002) for SPM5. Functional time series underwent a maximum overlay discrete wavelet transform as implemented by Achard et al. (2006) in the R-package Brainwaver (http://cran.r-project.org/web/packages/brainwaver), resulting in four frequency bands (level 1: $0.13-0.25 \mathrm{~Hz}$; level 2: 0.06-0.13 Hz; level 3: $0.03-0.06 \mathrm{~Hz}$; level 4: $0.01-0.03 \mathrm{~Hz}$ ) with frequency band-specific correlation matrices of the 90 regions (for the full analysis pipeline, see supplemental Fig. S1, available at www.jneurosci.org as supplemental material). As previous studies have shown that small-world properties of human functional brain networks are most robust at the frequency band $0.03-0.06 \mathrm{~Hz}$ (Achard et al., 2006), and magnetoencephalography and fMRI studies observed a peak frequency of spontaneous oscillations $<0.05-0.06 \mathrm{~Hz}$ (Cordes et al., 2001; Stam, 2004; Salvador et al., 2005), we report results regarding this frequency band of interest. Results on level 4 $(0.01-0.03 \mathrm{~Hz})$ can be found in supplemental Figure S2 (available at www.jneurosci.org as supplemental material). Note that as our epochs were relatively brief and consisted of 150 time points, the frequency band 0.01- $0.03 \mathrm{~Hz}$ yielded few data for reliable wavelet correlation estimations.

We performed an additional analysis using a low-pass filter $(<0.10$ $\mathrm{Hz}$ ) in FSL 4.0 (www.fmrib.ox.ac.uk/fsl) on the residual images. Extraction of the time courses occurred according to the same procedure as described above, and time courses were correlated with Pearson's $R$ correlation coefficient to obtain a $90 \times 90$ correlation matrix. The results of this analysis are reported in supplemental Figure S3 (available at www. jneurosci.org as supplemental material) and below.

Graph theoretical analysis of functional correlation matrices. The correlation matrices were thresholded to obtain a binary connection matrix that was used to calculate network properties as the clustering coefficient $C$ and the mean shortest path length $L$ with the Brain Connectivity Toolbox for Matlab (Rubinov and Sporns, 2010). Because there is no gold standard for a threshold for such a binary matrix (e.g., a connection probability of $10 \%$ ), we report binary connection matrices with all possible thresholds unless otherwise stated. The wavelet correlation threshold used to obtain a particular connection probability served as an indicator for general connectivity, and all Pearson's $R$ values were Fisher $z$-transformed to fulfill the assumption of normality for the parametric tests that are described in the subsection Statistical testing. At the 
lowest thresholds (e.g., connection probabilities of 10 and 20\%), nodes may be disconnected from the network, resulting in infinite path lengths. In line with Hayasaka and Laurienti (2010), we therefore calculated the path length $L$ as the harmonic mean of geodesic distances.

By definition (Watts and Strogatz, 1998), a network has a small-world organization if the ratio gamma $(\gamma)$, defined as $C / C_{\text {random }}$, is $>1$ and a ratio lambda $(\lambda)$, defined as $L / L_{\text {random }}$, is $\sim 1$, with $C_{\text {random }}$ and $L_{\text {random }}$ reflecting the clustering coefficient and path length of a random network with the same connection probability. As suggested by Stam et al. (2007), a random graph should have a similar degree distribution to the original graph. In line with previous research (Liu et al., 2008), we randomly rewired thresholded graphs according to a Markov chain (Maslov and Sneppen, 2002) as implemented in the BCT-toolbox (Rubinov and Sporns, 2010). Both $C_{\text {random }}$ and $L_{\text {random }}$ were calculated as the average clustering coefficient and path length of 10 randomly rewired matrices that were generated for each of the 92 epochs at each threshold. Smallworldness $(\sigma)$ was calculated as gamma/lambda, with values $>1$ reflecting a small-world topology.

Visualization of functional brain networks. Binary connection matrices were visualized using the Pajek software package (vlado.fmf.uni-lj.si/ $\mathrm{pub} /$ networks/pajek). For a multidimensional visualization of functional connectivity in different sleep stages the Kamada-Kawai layout algorithm was used (Kamada and Kawai, 1989), which locates highly connected nodes (network hubs) in the geometrical center and sparsely connected nodes more peripherally, based on their path length. This layout algorithm iteratively adjusts the positions and forces the nodes to minimize the total energy of the system. For this visualization, binary connection matrices were generated at a connection probability of $25 \%$ to create a network that was sparsely connected but not fragmented in S0. In addition, differential (anatomical) graphs were created to illustrate which correlation pairs had an absolute differential correlation $\mid \Delta \mathrm{R} \|>$ 0.20 between two stages.

Analysis of subsystems. To evaluate subsystem-specific connectivity changes throughout sleep, we categorized the 90 regions into five subsystems according to Mesulam (2000): primary sensory, association, subcortical, paralimbic, and limbic regions (see supplemental Table S2, available at www.jneurosci.org as supplemental material). The correlation coefficients were grouped into 1 of 15 possible categories (e.g., association-association, association-limbic, association-primary sensory), averaged per category and Fisher $z$-transformed to fulfill the assumption of normality for the parametric tests that are described under the subheading Statistical testing.

Connection length. To examine whether sleep alters the ratio of long versus short connections, we split all possible connections into two groups with distances $>75$ and $\leq 75 \mathrm{~mm}$ in length in MNI space between nodes (Achard et al., 2006). The length of a specific connection was calculated by subtracting the respective [XY Z] coordinates of two nodes, $i$ and $j$, from each other and square root transform the sum of the squared differential scores, as follows:

$$
\text { Length }=\sqrt{\left[\left(X_{i}-X_{j}\right)^{2}+\left(Y_{i}-Y_{j}\right)^{2}+\left(Z_{i}-Z_{j}\right)^{2}\right]} .
$$

We then calculated per epoch the mean ( $z$-transformed) correlation coefficient for the long distances and the short distances, and the ratio between both (long-short) correlation values was compared across sleep stages as described below.

Statistical testing. To test between-stage differences in network properties and correlations, we generated tables with correlation values and graph theoretical metrics for all 92 epochs at different thresholds (connection probabilities ranged from $10 \%$ to $90 \%$ with incremental steps of $10 \%)$. We included subject identification as an additional variable to account for nestedness of the data (e.g., a subject could have an epoch in several sleep stages) with linear mixed models as implemented in SPSS 16.0. We used a significance level of 0.001 to evaluate the main effect of sleep, and when the main effect was significant, the direction of the effect was evaluated with post hoc $t$ tests at a nominal $\alpha$. We used a Bonferronicorrected $\alpha$ to test the main effect of sleep on between- and withinsubsystem correlations (corrected for 15 correlations) and on differences in single regions such as the thalamus (corrected for 90 regions).
Power spectra. To evaluate whether the relative power in the frequency band of interest changed throughout sleep, we performed a fast Fourier transform on the regional time courses. We analyzed the power in the frequency band of interest $(0.03-0.06 \mathrm{~Hz})$ relative to the total power $(0.01-0.25 \mathrm{~Hz})$ for each epoch, and used this ratio in a linear mixed model to account for nestedness of the data as described above.

\section{Results \\ Correlation threshold values and small-world properties throughout sleep}

There was a significant effect of sleep stage on the wavelet threshold correlation at most thresholds (Fig. 1 A, Table 2), with post hoc tests revealing that sleep stages S1 and S2 had higher wavelet correlation values than both S0 and SW. There was also a significant effect of sleep stage on the clustering coefficient $C$ (Fig. $1 B$, Table 2), with post hoc tests showing the same direction (S1/S2 > $\mathrm{S} 0 / \mathrm{SW}$ ), although for several thresholds S0 had a value equal to S1 and $\mathrm{S} 2(\mathrm{~S} 0 / \mathrm{S} 1 / \mathrm{S} 2>\mathrm{SW})$. However, the $C_{\text {random }}$ values showed the same differences across sleep stages $(\mathrm{S} 1 / \mathrm{S} 2>\mathrm{S} 0 / \mathrm{SW})$, with even higher $F$ values for the main effect of sleep (Table 2). Therefore, the gamma $\left(C / C_{\text {random }}\right)$ values had the reverse direction: the main effect of sleep stage on gamma was significant at all thresholds, and post hoc tests revealed that $\mathrm{SW}>\mathrm{S} 0>\mathrm{S} 1 / \mathrm{S} 2$ or SW/ S0 $>$ S1/S2 (Table 2, Fig. 1C). The effect of sleep on path length $L$ and lambda $\left(L / L_{\text {random }}\right)$ was not significant except at a few of the lowest thresholds (Table 2, Fig. 1D,E). The effect of sleep on small-worldness $\sigma$ was significant, with post hoc tests revealing that $\mathrm{SW}>\mathrm{S} 0>\mathrm{S} 1 / \mathrm{S} 2$ or $\mathrm{SW} / \mathrm{S} 0>\mathrm{S} 1 / \mathrm{S} 2$; this effect was driven by gamma. Note that these changes across sleep stages were most robust for this frequency band $(0.03-0.06 \mathrm{~Hz}$ ) (for the frequency band $0.01-0.03 \mathrm{~Hz}$, see supplemental Fig. S2, available at www. jneurosci.org as supplemental material) and that a similar pattern could be observed in the analysis of the low-pass-filtered time series $(<0.10 \mathrm{~Hz})$ (see supplemental Fig. S3, available at www. jneurosci.org as supplemental material).

To examine the increased $C_{\text {random }}$ values in light sleep, we first evaluated the random rewiring process in a $K_{\text {nearest-neighbor }}$ versus $K$ plot of the average correlation matrices thresholded at a connection probability of $30 \%$ (supplemental Fig. S4, available at www.jneurosci.org as supplemental material). This plot shows that the $K_{\text {nearest-neighbor }}$ of a node is independent from its $K$, as expected in a random graph. We subsequently examined the $C$ versus $K$ plots at the same threshold, and these illustrated that although the $C$ values are generally higher in S1 and S2, so are the

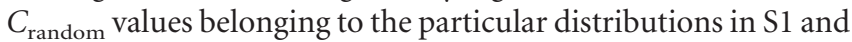
S2 (supplemental Fig. S5, available at www.jneurosci.org as supplemental material). Histograms of the sleep-specific degree distributions (supplemental Fig. S6, available at www.jneurosci.org as supplemental material) demonstrated that S1 and S2 have a flatter distribution than S0 and SW. To compare these sleep stage-specific distributions with a flat distribution with equal frequencies per category, we recoded $K$ into four categories (see legend to supplemental Fig. S6, available at www.jneurosci.org as supplemental material), and we observed that S1 and S2 did not significantly differ from a flat distribution $\left[\chi^{2}(3)=4.4, p=0.221\right.$ and $\chi^{2}(3)=3.6, p=0.308$, respectively], whereas S0 and SW did $\left[\chi^{2}(3)=19.4, p<0.001\right.$ and $\left.\chi^{2}(3)=37.8, p<0.001\right]$.

\section{Subsystem connectivity throughout sleep}

The differences in general connectivity across sleep stages were significant in connections involving association, primary sensory, and paralimbic cortices (for Fisher $z$-transformed correlation values, see Table 3). Post hoc tests revealed that correlations 
A

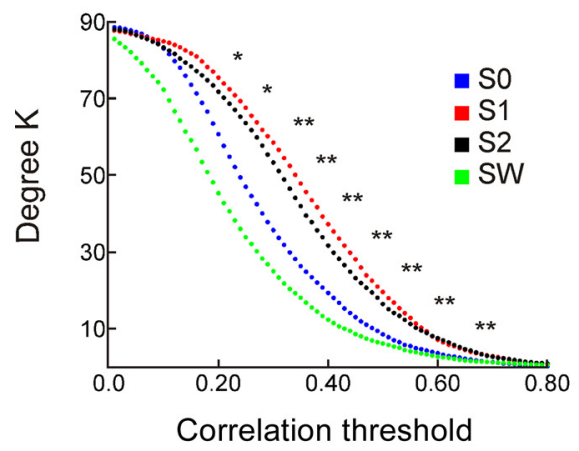

D

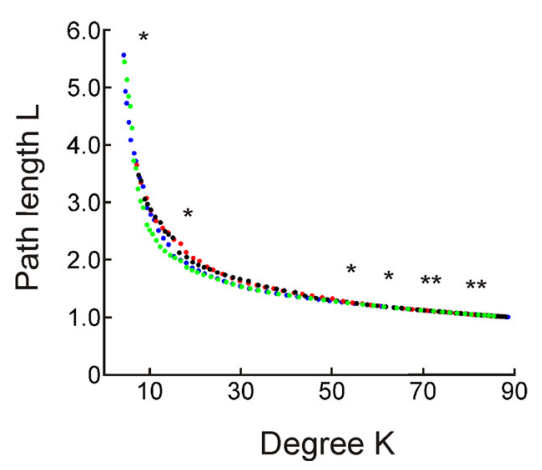

B

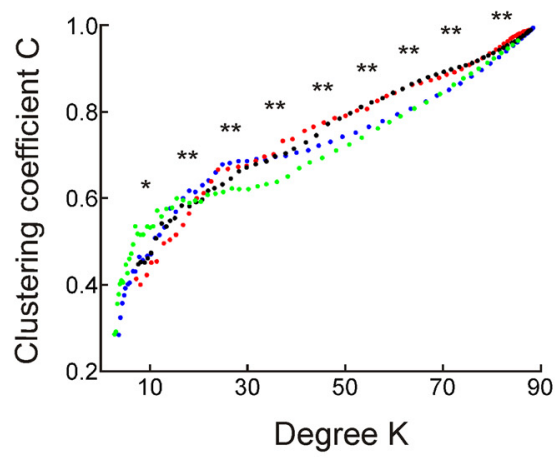

E

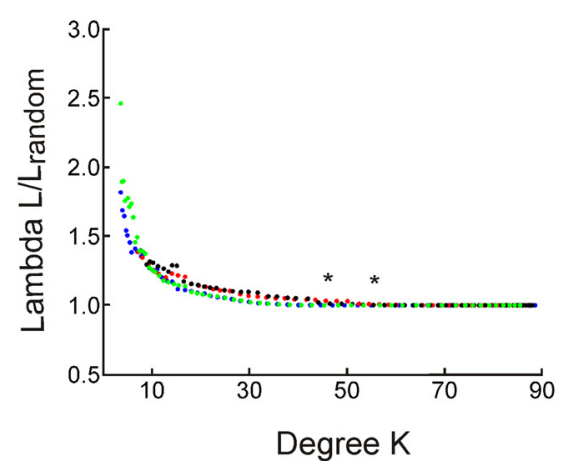

C

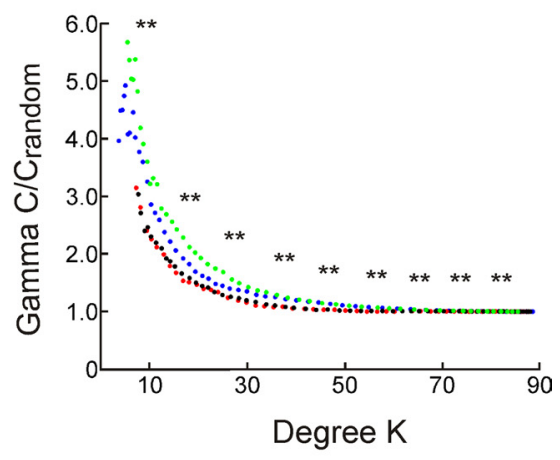

F

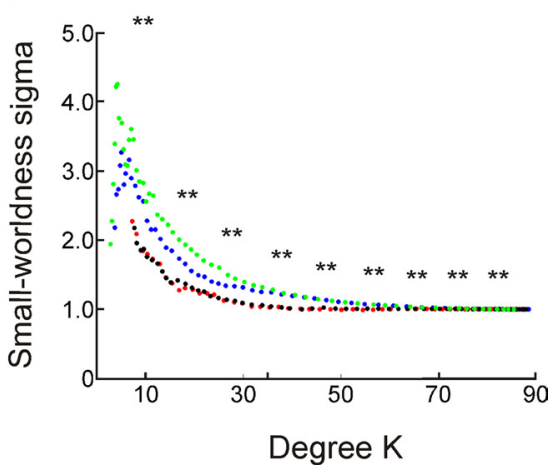

Figure 1. Connectivity and small-world metrics throughout sleep at various thresholds, depicting the wavelet correlation threshold, clustering coefficient $(C)$, gamma $\left(C / C_{\text {random }}\right)$, path length $(L)$, lambda $\left(L / L_{\text {random }}\right)$, and small-worldness $\sigma$ (gamma/lambda) at all possible thresholds for SO, S1, S2, and SW, for the frequency band $0.03-0.06 \mathrm{~Hz}$. $A$, There was a significant effect of sleep on $R_{\text {thresh }}$, with post hoc tests indicating that values were higher in S1/S2 than in SO/SW (Table 1). B, A significant effect of sleep on C is shown, with post hoc tests revealing higher values in S1/S2 than in SO/SW. C, Shows the reverse pattern for gamma as $C_{\text {random }}$ values were also highest in light sleep. $\boldsymbol{D}, \boldsymbol{E}$, The effect of sleep on $L$ and lambda was not robustly significant. $\boldsymbol{F}$, The significant effect of sleep on sigma (driven by gamma) is depicted, with post hoc tests revealing higher values in S0/SW than in S1/S2, or intermediate values for S0. ${ }^{* *}$ Significant at 0.001 ; ${ }^{*}$ significant at 0.05 .

Table 2. Differences in small-world metrics across sleep stages at different thresholds (mean degree $K /$ connection probability)

\begin{tabular}{|c|c|c|c|c|c|c|c|c|}
\hline K & Correlation & $C$ & $C_{\text {random }}$ & $L$ & $L_{\text {random }}$ & $C / C_{\text {random }}$ & $L / L_{\text {random }}$ & Sigma \\
\hline $9(10 \%)$ & $9.8(86.0)^{* *}$ & $2.7(82.0)$ & $5.7(83.3)^{*}$ & $4.8(84.2)^{*}$ & $5.4(84.2)^{*}$ & $7.7(83.3)^{* *}$ & 1.7 (81.2) & $7.9(83.7)^{* *}$ \\
\hline $18(20 \%)$ & $8.9(86.1)^{* *}$ & $0.7(86.2)$ & $8.4(84.8)^{* *}$ & $3.8(85.6)^{*}$ & $4.1(85.2)^{*}$ & $8.0(84.8)^{* *}$ & $2.5(84.6)$ & $8.7(84.9)^{* *}$ \\
\hline $27(30 \%)$ & $8.3(86.1)^{* *}$ & $8.0(86.3)^{* *}$ & $10.1(85.0)^{* *}$ & $1.3(84.8)$ & $0.9(83.6)$ & $8.1(83.9)^{* *}$ & $2.2(85.3)$ & $8.5(84.1)^{* *}$ \\
\hline $36(40 \%)$ & $7.9(86.1)^{* *}$ & $11.0(86.3)^{* *}$ & $10.4(85.4)^{* *}$ & $0.3(83.9)$ & $1.7(84.0)$ & $8.3(83.3)^{* * *}$ & $1.4(85.5)$ & $8.4(83.8)^{* *}$ \\
\hline $45(50 \%)$ & $7.5(86.1)^{* *}$ & $9.5(86.2)^{* *}$ & $10.7(85.6)^{* *}$ & $2.2(84.2)$ & $5.1(85.5)^{*}$ & $9.4(83.7)^{* *}$ & $2.8(85.6)^{*}$ & $9.7(84.1)^{* *}$ \\
\hline $54(60 \%)$ & $7.3(86.1)^{* *}$ & $9.3(86.2)^{* *}$ & $10.7(85.7)^{* *}$ & $4.5(85.6)^{*}$ & $6.3(85.6)^{*}$ & $9.9(84.1)^{* *}$ & $3.1(86.1)^{*}$ & $10.3(84.2)^{* *}$ \\
\hline $63(70 \%)$ & $6.8(86.1)^{* *}$ & $9.3(86.0)^{* *}$ & $10.5(85.7)^{* *}$ & $6.0(85.6)^{*}$ & $6.8(85.7)^{* *}$ & $9.7(84.0)^{* *}$ & $1.8(88.0)$ & $10.0(84.0)^{* *}$ \\
\hline $72(80 \%)$ & $5.9(86.1)^{*}$ & $9.4(85.9)^{* *}$ & $10.0(85.7)^{* *}$ & $6.6(85.6)^{* *}$ & $6.9(85.6)^{* *}$ & $8.9(84.3)^{* *}$ & $1.2(88.0)$ & $9.2(84.3)^{* *}$ \\
\hline $81(90 \%)$ & $4.4(86.1)^{*}$ & $9.0(85.7)^{* *}$ & $9.3(85.7)^{* *}$ & $6.9(85.5)^{* *}$ & $6.9(85.5)^{* *}$ & $8.4(84.2)^{* *}$ & $2.0(88.0)$ & $8.5(84.2)^{* *}$ \\
\hline Post hoc ${ }^{a}$ & $\begin{array}{l}\mathrm{S} 1 / \mathrm{S} 2>\mathrm{SO} / \mathrm{SW} \\
(\mathrm{S} 1 / \mathrm{S} 2>\mathrm{S} 0>\mathrm{SW})\end{array}$ & $\begin{array}{l}\mathrm{S} 1 / \mathrm{S} 2>\mathrm{SO} / \mathrm{SW} \\
(\mathrm{SO} / \mathrm{S} 1 / \mathrm{S} 2>\mathrm{SW})\end{array}$ & $\begin{array}{l}\mathrm{S} 1 / \mathrm{S} 2>\mathrm{SO}>\mathrm{SW} \\
(\mathrm{S} 1 / \mathrm{S} 2>\mathrm{SO} / \mathrm{SW})\end{array}$ & $\mathrm{S} 1>\mathrm{SO} / \mathrm{SW}$ & $\begin{array}{l}\text { SO/SW }>S 1 / S 2 \\
(\mathrm{SW}>\mathrm{S} 1 / \mathrm{S} 2)\end{array}$ & $\begin{array}{l}\mathrm{SW}>\mathrm{SO}>\mathrm{S} 1 / \mathrm{S} 2 \\
(\mathrm{SO} / \mathrm{SW}>\mathrm{S} 1 / \mathrm{S} 2)\end{array}$ & $\mathrm{S} 2>\mathrm{SO} / \mathrm{SW}$ & $\begin{array}{l}\mathrm{SW}>\mathrm{SO}>\mathrm{S} 1 / \mathrm{S} 2 \\
(\mathrm{SO} / \mathrm{SW}>\mathrm{S} 1 / \mathrm{S} 2)\end{array}$ \\
\hline
\end{tabular}

$F$ values of the main effect of sleep are provided with the adapted $d f_{2}$ between parentheses $\left(d f_{1}=3\right)$.

${ }^{a}$ Post hoc test results at most thresholds; alternative (and less frequent) results are between parentheses.

${ }^{*} p<0.05 ;{ }^{* *} p<0.001$.

were highest in S1 and S2, and lowest in SW, with S0 taking an intermediate position for some subsystem correlations. Betweensleep-stage differences were insignificant or nominally significant when the correlation pair included either the subcortical or the limbic subsystem, with one exception (primary sensory-limbic).

\section{Differential connectivity between sleep stages}

Between-stage differential connectivity graphs are displayed in Figure 2 and involved nodes are described in supplemental Table S3 (available at www.jneurosci.org as supplemental material). In the contrast $\mathrm{S} 0>\mathrm{S} 1$, the differential correlations were limited to the bilateral thalamus: of a total of 89 pairwise correlations, the left thalamus had a $\Delta R>0.20$ in 62 correlations and the right thalamus in 46 correlations. As an illustration of how dissimilar this differential connectivity of the thalamus was to other regions, the region with the third-strongest differential connectivity from S0 to $\mathrm{S} 1$ (superior temporal gyrus) had a $\Delta R>0.20$ in only three pairwise correlations. In S2 and SW, these correlations were partially restored: the left thalamus had 50 and the right thalamus 22 correlations with a $\Delta R>0.20$ in the contrast $\mathrm{SW}>\mathrm{S} 1$ (see supplemental Fig. S2, available at www.jneurosci.org as supplemental material). A linear mixed models ANOVA showed that there was a significant effect of sleep on mean Fisher $z$-values of the left thalamus $\left(F_{(3,84.5)}=6.75, p<0.05 / 90 \sim 0.0006\right)$ and on mean Fisher $z$-values of the right thalamus $\left(F_{(3,83.9)}=6.45, p<\right.$ $0.0006)$, with the latter threshold reflecting an $\alpha$ that was Bonfer- 
Table 3. Between sleep-stage differences in subsystem connectivity tested by linear mixed models on the Fisher $z$-transformed correlation coefficients $\left(d f_{1}=3\right.$ )

\begin{tabular}{|c|c|c|c|c|}
\hline Subsystems & $F$ & $\mathrm{df}_{2}$ & $p$ & Posthoc $(p<0.05)$ \\
\hline \multicolumn{5}{|l|}{ Neocortical connections } \\
\hline Association-Association & 9.15 & 86.0 & $<0.001^{* *}$ & $\mathrm{~S} 1 / \mathrm{S} 2>\mathrm{SO}>\mathrm{SW}$ \\
\hline Association-Primary & 11.16 & 85.7 & $<0.001^{* *}$ & $\mathrm{~S} 1 / \mathrm{S} 2>\mathrm{SO}>\mathrm{SW}$ \\
\hline Primary-Primary & 7.69 & 85.2 & $<0.001^{* *}$ & $\mathrm{~S} 1 / \mathrm{S} 2>\mathrm{SO} / \mathrm{SW}$ \\
\hline \multicolumn{5}{|c|}{ Neocortical/(para)limbic connections } \\
\hline Association-Paralimbic & 11.82 & 85.2 & $<0.001^{* *}$ & $\mathrm{~S} 1 / \mathrm{S} 2>\mathrm{S} 0 / \mathrm{SW}$ \\
\hline Association-Limbic & 2.42 & 85.6 & 0.072 & \\
\hline Limbic-Primary & 4.94 & 83.4 & $0.003^{* *}$ & $\mathrm{~S} 1>\mathrm{S} 2>\mathrm{SW}, \mathrm{SO}>\mathrm{SW}$ \\
\hline Paralimbic-Primary & 15.06 & 85.7 & $<0.001^{* *}$ & $\mathrm{~S} 1 / \mathrm{S} 2>\mathrm{S} 0 / \mathrm{SW}$ \\
\hline \multicolumn{5}{|c|}{ Limbic/paralimbic connections } \\
\hline Limbic-Limbic & 0.61 & 84.7 & 0.611 & \\
\hline Limbic-Paralimbic & 1.95 & 85.7 & 0.127 & \\
\hline Paralimbic-Paralimbic & 6.76 & 84.5 & $<0.001^{* *}$ & $\mathrm{~S} 1 / 2>\mathrm{SO} / \mathrm{W}$ \\
\hline \multicolumn{5}{|c|}{ Connections including the subcortical subsystem } \\
\hline Association-Subcortical & 2.76 & 88.0 & $0.047^{*}$ & $(\mathrm{SO}>\mathrm{S} 2 / \mathrm{W})$ \\
\hline Limbic-Subcortical & 0.30 & 86.1 & 0.826 & \\
\hline Paralimbic-Subcortical & 1.35 & 88.0 & 0.263 & \\
\hline Primary-Subcortical & 1.33 & 82.6 & 0.272 & \\
\hline Subcortical-Subcortical & 0.79 & 84.4 & 0.505 & \\
\hline
\end{tabular}

*Significant at nominal $\alpha$ of 0.05 ; ** significant at Bonferroni-adapted $\alpha$ of $0.05 / 15=0.0033$.

roni corrected for 90 regions. Post hoc tests revealed that for the bilateral thalamus values were higher in S0 than in S1/S2/SW.

The increase in connectivity in S1 and S2 was mostly confined to primary sensory and association cortices (see supplemental Table S3 and supplemental Figs. S7-S9, available at www. jneurosci.org as supplemental material), with subcortical regions and angular and cingulate gyri as notable exceptions. The reduction in connectivity in SW additionally affected the hippocampus and adjacent regions. Several regions in the temporal lobe (hippocampus, parahippocampal gyrus, middle temporal gyrus, and temporal pole) had more positive correlations in S1 than SW (see supplemental Fig. S7, available at www.jneurosci.org as supplemental material). Differences in organization of the large-scale functional brain network are depicted in Figure 3, in which the thalamus is disconnected from the network in S1 and S2. Note the expansion of the network in association, primary sensory, and paralimbic cortices in SW.

\section{Decrease in the ratio long versus short distance correlations throughout sleep}

There was a significant effect of sleep on the $z$-transformed correlation values of short connections $(<75 \mathrm{~mm})\left(F_{(3,86.3)}=7.45\right.$, $p<0.001)$, and post hoc tests revealed that values were higher in S1/S2 than in S0/SW. In addition, we observed a similar effect of sleep on the $z$-transformed correlation values of long connections $(>75 \mathrm{~mm})\left(F_{(3,86.0)}=8.67, p<0.001\right)$, with post hoc tests again revealing that values were higher in S1/S2 than in S0/SW. There was a trend for a significant post hoc difference between $\mathrm{S} 0$ and SW regarding long-connection correlation values ( $p=0.090)$, which reflected that correlation values of long connections were more strongly reduced in SW than short connections. When we tested this with the ratio of long versus short $z$-transformed correlation values, we noted a significant effect of sleep $\left(F_{(3,85.2)}=\right.$ $4.11, p=0.009$ ), with post hoc tests showing that this ratio (long/ short) was lower in SW than in S0/S1/S2 (all $p<0.022$ ). There were no differences in this ratio between $\mathrm{S} 0$ and $\mathrm{S} 1, \mathrm{~S} 1$ and $\mathrm{S} 2$, or S0 and S2 (all $p>0.330)$.

\section{Differences in relative power across sleep stages}

There were few changes in the relative power (frequency band, $0.03-0.06 \mathrm{~Hz}$ relative to $0.01-0.25 \mathrm{~Hz}$ ) in any of the 90 tested regions, with the only nominally significant changes occurring in the left caudate and putamen and right supramarginal gyrus $(p<$ $0.05, \mathrm{SW}>\mathrm{S} 0 / \mathrm{S} 1 / \mathrm{S} 2$ ) (see supplemental Table S1, available at www.jneurosci.org as supplemental material). These differences were not significant at an $\alpha$ that was Bonferroni corrected for 90 regions. Note that there were no differences in absolute power for the frequency band $0.03-0.06 \mathrm{~Hz}$ between S0 and S1 for the left thalamus $\left(t_{(48)}=0.98, p=0.334\right)$, or for the right thalamus $\left(t_{(48)}=\right.$ $0.83, p=0.412)$.

\section{Discussion}

We observed that in the transition from wakefulness to light sleep stage S1, there was an increase in the correlation threshold to generate a network with a given connection probability. Moreover, the thalamus disconnected from the large-scale functional brain network at sleep onset. In the descent from light to slowwave sleep, corticocortical functional connectivity decreased, and there was a more pronounced reduction in the correlation strength of long than of short connections. In general, functional connectivity was highest in light sleep and lowest in slow-wave sleep, with wakefulness taking an intermediate position at some thresholds. There was also a significant effect of sleep on smallworld properties, in particular on local clustering values, which were highest in slow-wave sleep and lowest in light sleep when compared with values of random networks. This indicates that the large-scale functional brain network moves toward randomness in light sleep, whereas the reduced long-range connectivity and increased local clustering in slow-wave sleep are indicative of a shift toward a regular network (Watts and Strogatz, 1998), although a small-world topology $(\sigma>1)$ was present in all stages.

\section{Corticocortical connectivity throughout sleep}

We observed a widespread increase in corticocortical connectivity in light sleep stages (S1/S2, compared with wakefulness), but a strong reduction of corticocortical connectivity in slow-wave sleep. These findings extend prior simultaneous EEG/fMRI studies on BOLD signal fluctuations during sleep, which reported that functional connectivity was maintained in light sleep and broke down in slow-wave sleep. Horovitz et al. (2008) noted an increase in BOLD signal fluctuations in the visual cortices, auditory cortices, and the precuneus, among others, and that default mode 
network connectivity persisted in light sleep. Sämann et al. (2009) noted the continued presence of the default mode network in light sleep with a trendwise decrease in intranetwork connectivity. Larson-Prior et al. (2009) reported no measurable changes in functional connectivity in various sensory and cognitive resting networks from wakefulness to light sleep, while an increase in functional connectivity was observed in the dorsal attention network. In slow-wave sleep, a breakdown of the correlation between the anterior and posterior nodes of the default mode network was observed (Horovitz et al., 2009; Sämann et al., 2009), with the posterior areas maintaining or even strengthening their connectivity (Horovitz et al., 2009).

Our results extend these previous reports by showing that in particular primary sensory and association cortices showed sleep stage-specific differences with an increase in light sleep, and that the reduction in connectivity in slow-wave sleep was most pronounced in long-range connections. This breakdown of corticocortical and longrange connectivity in slow-wave sleep parallels a combined high-density EEG and transcranial magnetic stimulation (TMS) study during sleep (Massimini et al., 2005), which reported that TMS-induced activation spread over the cortex during wakefulness but remained local in slow-wave sleep. The focal pattern of changes in connectivity across sleep stages needs further attention, as we observed a more robust reduction of functional connectivity in slow-wave sleep in occipital, temporal, and parietal cortices than in prefrontal cortices. Furthermore, the increase in corticocortical connectivity in light sleep did not occur in all regions; notable exceptions were the subcortical regions and the angular and cingulate gyri.

\section{Thalamocortical connectivity throughout sleep}

We observed that there was one bilateral region with a higher connectivity in wakefulness than in light sleep: the thalamus. This shows that the paradoxical increase in functional connectivity in cortical regions in light sleep is accompanied by exclusion of a pivotal node from the thalamocortical network, showing that the fading of consciousness in light sleep has a temporal relationship with reduced thalamocortical (but not corticocortical) connectivity. This is in line with the information integration theory of consciousness (Tononi, 2004), which states that not connectivity per se but rather the capacity to integrate information across different modules determines the fading of consciousness in early NREM sleep (Tononi and Massimini, 2008).

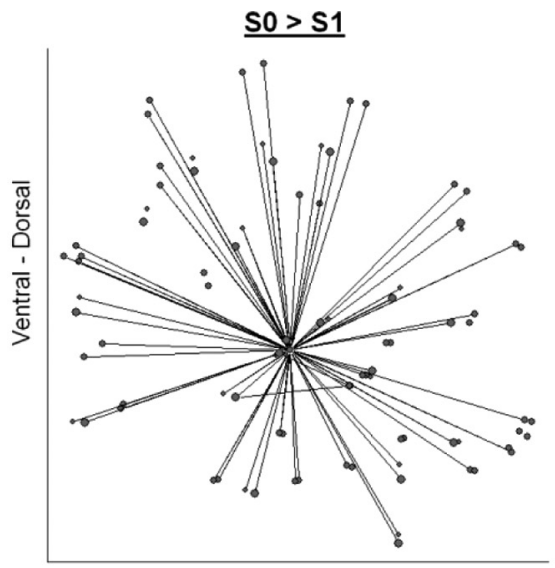

Posterior - Anterior

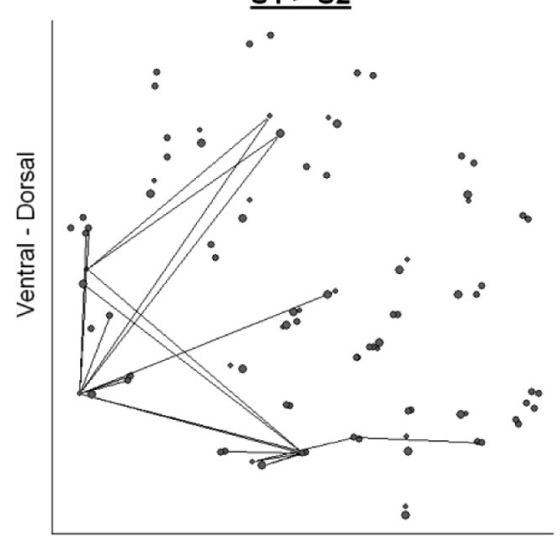

Posterior - Anterior

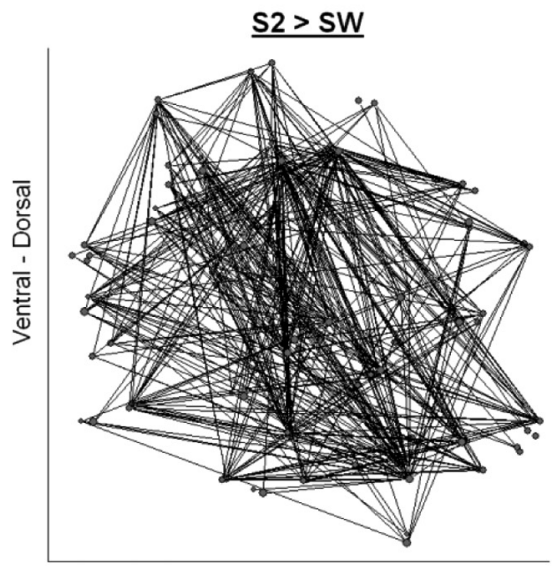

Posterior - Anterior
$\underline{\mathrm{S1}>\mathrm{S2}}$

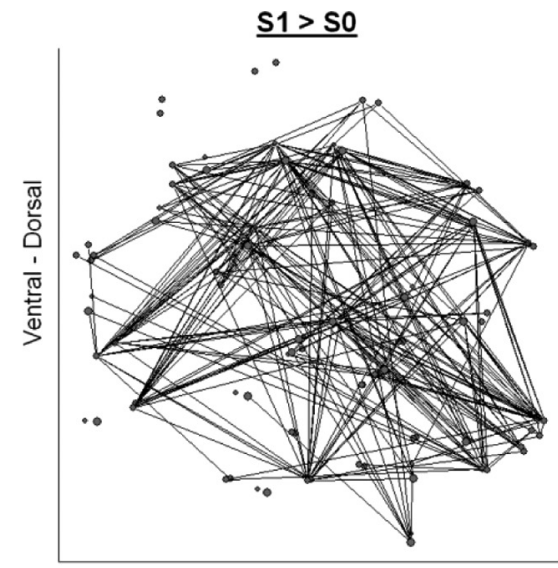

Posterior - Anterior

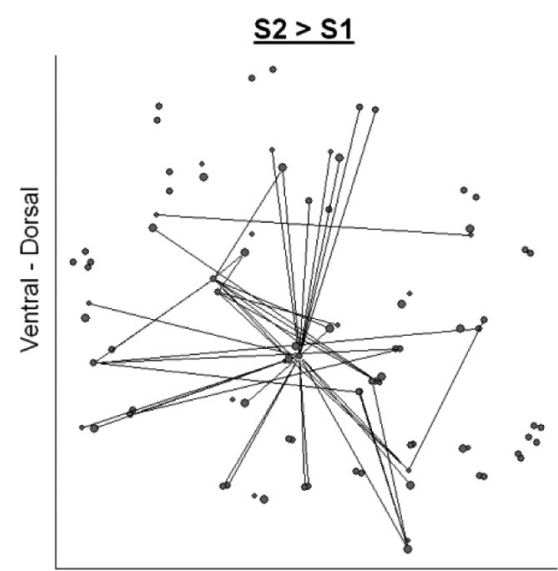

Posterior - Anterior

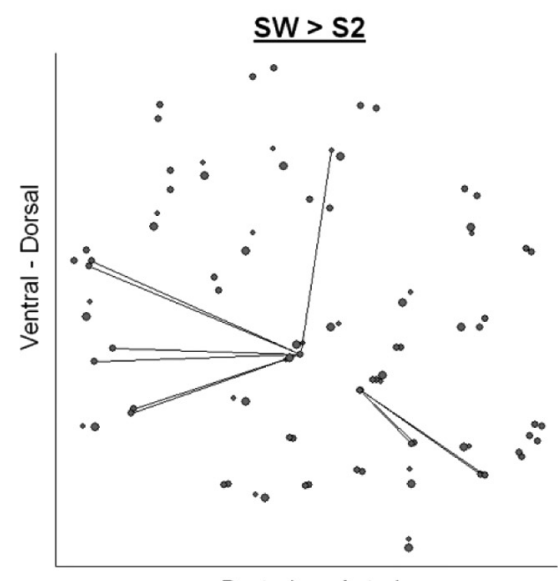

Posterior - Anterior
Figure 2. Differential connectivity graphs. Figure 2 illustrates differences in connections of 90 cortical and subcortical regions in the transition from $S 0$ to $S 1$ and $S 2$, and finally to $S W$, with lines depicting a $\mid \Delta R \|>0.20$ between two stages. Top left, lllustrates the breakdown of thalamocortical connectivity from $\mathrm{SO}$ to $\mathrm{S} 1$, whereas the top right displays the increased corticocortical connectivity in $\mathrm{S} 1$ compared with $\mathrm{S} 0$. There are few differences between $\mathrm{S} 1$ and $\mathrm{S2}$, whereas a reduction in corticocortical connectivity in SW is depicted in the lower left panel (for the other contrasts, see supplemental Fig. S7, available at www.jneurosci.org as supplemental material; regions with at least 15 differential connections are described in supplemental Table $\mathbf{S} 3$ available at www.jneurosci.org as supplemental material). Note that the current threshold of $\mid \Delta R \|>0.20$ is an arbitrary threshold as $z$-values for correlation differences fell between $[-1.5 ; 1.5]$ (see supplemental Figs. $\$ 8$ and S9, available at www.jneurosci.org as supplemental material) and were not significant in univariate tests due to modest group sizes. Figure 2 is provided as an illustration of between-stage connectivity differences that are described in Table 3 or in the Results section.

The reduction in thalamocortical correlations was specific to NREM sleep stage 1, as there was a trend for partial restoration of thalamocortical connectivity in sleep stage 2 and slow-wave sleep. Sleep stage 1 is considered a transition period rather than a sleep 


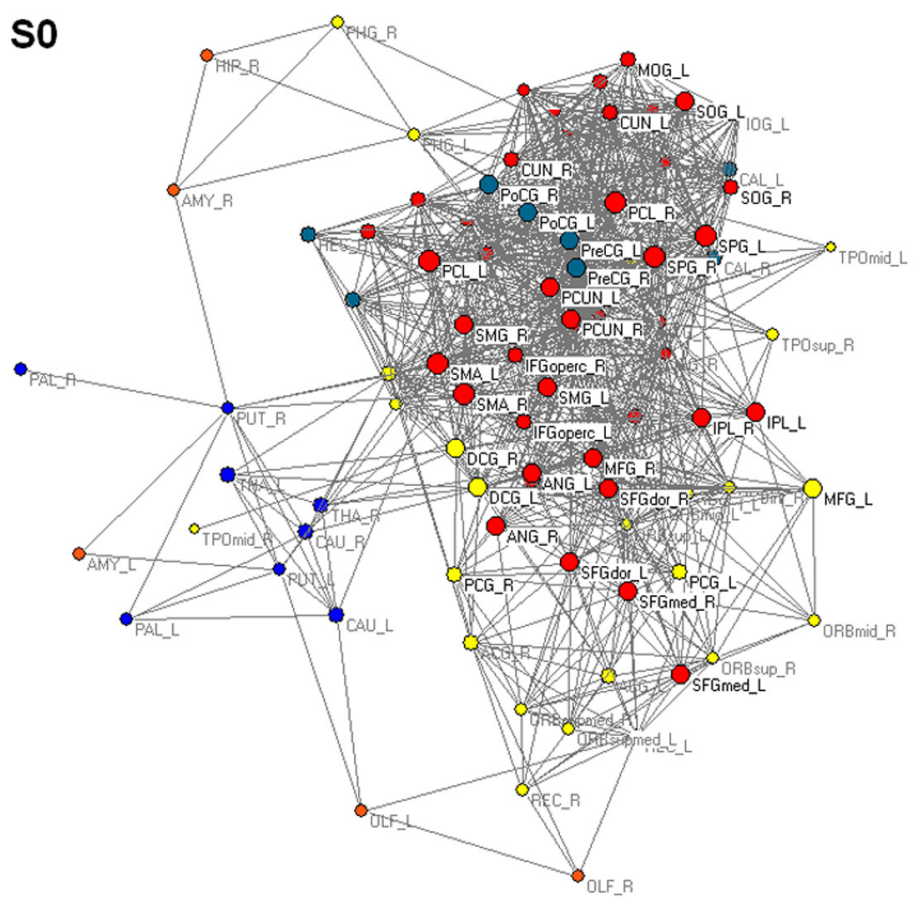

S1

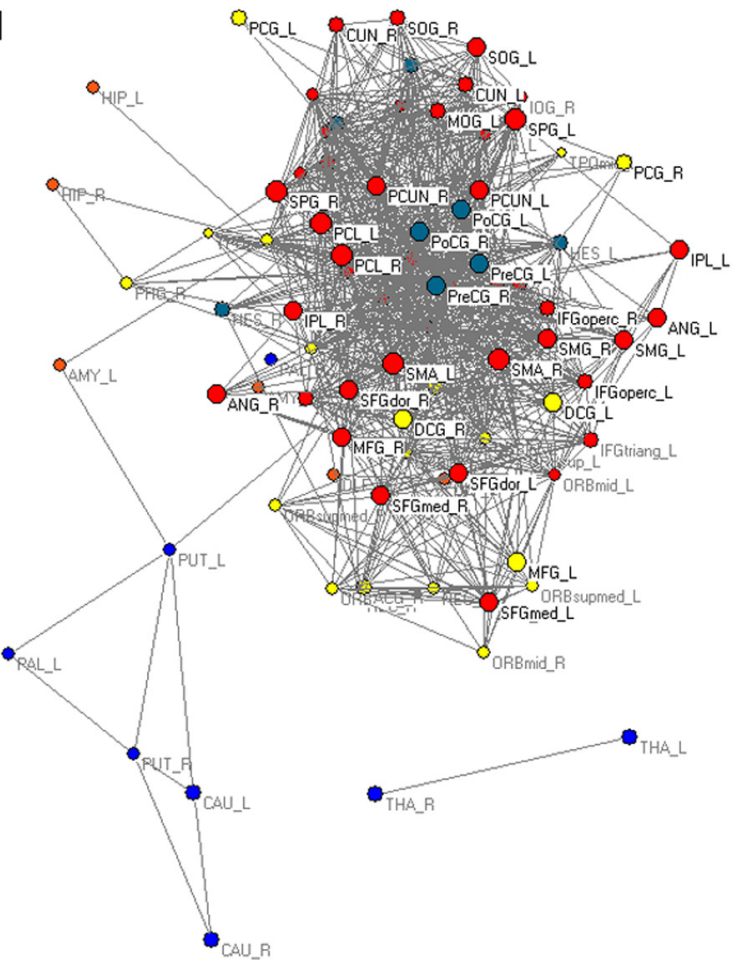

S2

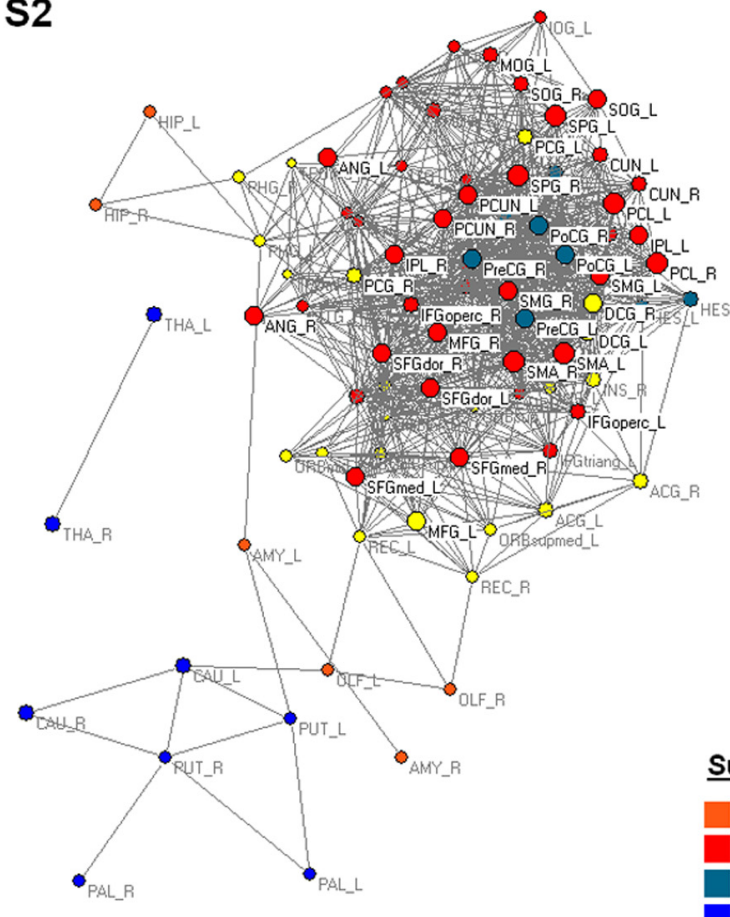

Subnetworks

Limbic

Association

Primary

Subcortical

Paralimbic sw

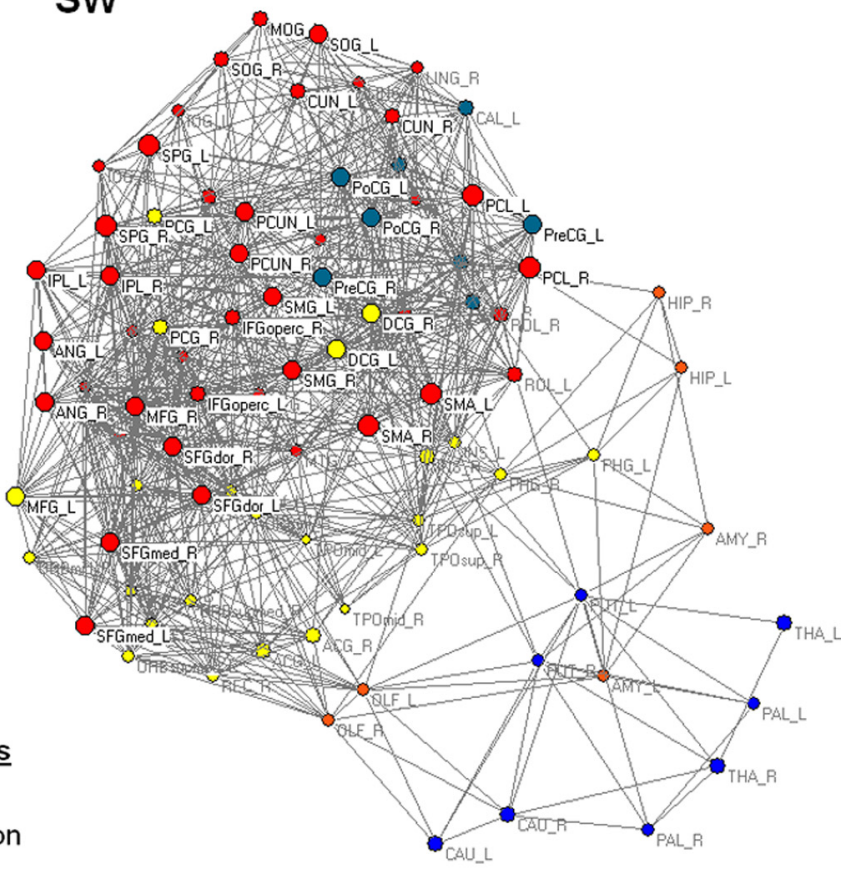

Figure 3. Algorithmic graphs of wakefulness and each NREM sleep stage. The networks represent binary connections, locally organized by a layout algorithm (Kamada and Kawai, 1989) implemented in the Pajek software package. The algorithm draws distances between nodes as a function of path length. Well-connected nodes (hubs) appear more central, whereas more isolated regions are located in the periphery. This algorithm further iteratively adjusts the positions of nodes and forces them to reduce the total energy of the system to a minimum (for labels, see supplemental Table S2, available at www.jneurosci.org as supplemental material). For this visualization, binary connection matrices were generated at a connection probability of $25 \%$ to create a network that was sparsely connected but not fragmented in SO.

stage per se by several authors (Johnson et al., 1976; Ogilvie et al., 1991), with sleep stage 2 reflecting more consolidated sleep. Auditory target detection is higher in sleep stage 1 than stage 2 (Campbell and Colrain, 2002; Cote et al., 2002), although waking detection levels are much higher (Cote et al., 2002). Here, the subclassification of stage 1 into early stage 1 sleep (stage 1a) and later stage 1 sleep (stage 1b) may be informative, as detection levels in late stage 1 sleep appear reduced to levels typical for consolidated sleep (Niiyama et al., 1994). With our data, we can conclude that the breakdown of thalamocortical connectivity as 
measured by BOLD signal fluctuations has a temporal relationship with falling asleep but not with the consolidation or the deepening of sleep in later sleep stages. Partial restoration of thalamocortical connectivity in later sleep stages could be a consequence of sleep-specific phenomena originating in the thalamus, such as sleep spindles (Steriade et al., 1994).

\section{Small-world properties throughout sleep}

Achard et al. (2006) observed that small-world properties of a large-scale functional brain network with 90 nodes were most robust for the $0.03-0.06 \mathrm{~Hz}$ frequency band. We analyzed this frequency band of interest throughout sleep and observed a main effect of sleep on local clustering but not characteristic path length, which was also noted in studies on small-world properties in sleep that analyzed EEG synchronization (Ferri et al., 2007, 2008; Dimitriadis et al., 2009). In line with Ferri et al. (2008), we found that the clustering coefficient was increased in light sleep; however, in our data the clustering coefficients in light sleep were at the same time closest to clustering coefficients of randomly rewired graphs. The ratio gamma $\left(C / C_{\text {random }}\right)$ was therefore lowest in light sleep stages and highest in wakefulness and slow-wave sleep. This finding shows that the increase in corticocortical connectivity in light sleep was accompanied by a network organization that moved toward randomness. As Ferri et al. (2008) observed, the reverse direction for gamma values (highest in light sleep) at delta and alpha frequency bands, the frequency band specificity of differences in small-world properties throughout sleep requires further study.

We further observed an increase in local clustering compared with random values in slow-wave sleep, with higher values for gamma in slow-wave sleep than in wakefulness at several thresholds. Although there were no significant differences for characteristic path length or its ratio lambda, the correlation values of physical long-distance connections were sharply reduced in slow-wave sleep. As a result, the large-scale functional brain network in slow-wave sleep demonstrated both high "cliqueness" and fewer physical long-range connections, which reflects a move toward a regular network (Watts and Strogatz, 1998). This network organization provides a functional brain state optimal for reprocessing information in segregated functional systems, which is in accord with a memory reprocessing hypothesis of slow-wave sleep (Diekelmann and Born, 2010). The functional relevance of a shift of the large-scale low-frequency functional brain network to more randomness in light sleep, and to more regularity in slow-wave sleep, needs further attention, as network reconfiguration in sleep may well be a key mechanism for our understanding of plasticity taking place during sleep (Ferri et al., 2008).

\section{Methodological considerations}

The advantage of fMRI over EEG is that connectivity of subcortical structures can be evaluated, but the poor temporal resolution of fMRI places a restriction on the frequency bands that can be examined. Moreover, the temporal resolution of fMRI requires long epochs for a reliable estimation of functional connectivity. In our study, we used 300 s epochs containing 150 time points, an epoch length that is not uncommon for analyses of resting-state functional connectivity, albeit relatively short. Longer time series would lead to better estimates of functional connectivity (Bullmore et al., 2004), manifest in smaller confidence intervals of wavelet correlations (relevant for the frequency band $0.01-0.03 \mathrm{~Hz}$ ). However, these have the practical difficulty that sleep measurements in the MRI scanner rarely contain pro- longed intervals of one continuous sleep stage. The noise and uncomfortable sleeping position increase the chance of transitions between sleep stages (and wakefulness). Our analysis is therefore a result of optimizing the balance between epoch length and epoch amount for analysis.

During preprocessing, we controlled for nuisance factors that may inflate wavelet correlations of fMRI time series (Bullmore et al., 2004) by regressing out movement, white matter, and CSF fluctuations and extracting time courses of regions of interest from the residual images. In a comparison of several preprocessing strategies, Weissenbacher et al. (2009) reported that the specificity of positive resting-state correlations is optimal when, in addition, global signal changes are removed. We refrained from this option as the global signal is highly correlated with the thalamus and was therefore excluded from previous studies on thalamocortical connectivity (Zhang et al., 2008), and since controlling for the global signal also generates artificial anticorrelations that may hamper interpretation of graph theory analyses.

Anatomical parcellation of the supratentorial brain compartment was performed using the Automated Anatomic Labeling (AAL) atlas (Tzourio-Mazoyer et al., 2002). We used this regionbased approach (Achard et al., 2006; He et al., 2007; Bassett et al., 2008; Supekar et al., 2009; Wang et al., 2009) instead of a voxelwise approach (Eguíluz et al., 2005; van den Heuvel et al., 2008), although voxelwise approaches provide enhanced spatial localization and increased small-world properties (Hayasaka and Laurienti, 2010). Since the AAL scheme is based on conventional macroscopic anatomical landmarks, it does not necessarily reflect the functional organization of the brain, as for instance that derived from resting state network analyses (Fox and Raichle, 2007). This may affect some of the bivariate connections reported here, possibly leading to an underestimation of functional connectivity. Since we aimed to examine differences in smallworldness across sleep stages, we chose the more conservative region-based approach.

\section{Conclusion}

Our study indicated that graph theory and connectivity metrics provide a promising framework to describe functional dynamics across different sleep stages. Our results may be relevant to the debate over whether low-frequency BOLD signal fluctuations allow us to describe changes in consciousness (He and Raichle, 2009; Koch, 2009) and underline the important role of intrinsic human brain organization in such dynamic phenomena as sleep.

\section{References}

Achard S, Salvador R, Whitcher B, Suckling J, Bullmore E (2006) A resilient, low-frequency, small-world human brain functional network with highly connected association cortical hubs. J Neurosci 26:63-72.

Bassett DS, Bullmore E (2006) Small-world brain networks. Neuroscientist 12:512-523.

Bassett DS, Bullmore E, Verchinski BA, Mattay VS, Weinberger DR, MeyerLindenberg A (2008) Hierarchical organization of human cortical networks in health and schizophrenia. J Neurosci 28:9239-9248.

Brett M, Anton JL, Valabregue R, Poline JB (2002) Region of interest analysis using an SPM toolbox. Paper presented at 8th International Conference on Functional Mapping of the Human Brain, Sendai, Japan, June.

Bullmore E, Sporns O (2009) Complex brain networks: graph theoretical analysis of structural and functional systems. Nat Rev Neurosci 10:186-198.

Bullmore E, Fadili J, Maxim V, Sendur L, Whitcher B, Suckling J, Brammer M, Breakspear M (2004) Wavelets and functional magnetic resonance imaging of the human brain. Neuroimage 23 [Suppl 1]:S234-S249.

Campbell KB, Colrain IM (2002) Event-related potential measures of the inhibition of information processing: II. The sleep onset period. Int J Psychophysiol 46:197-214. 
Cordes D, Haughton VM, Arfanakis K, Carew JD, Turski PA, Moritz CH, Quigley MA, Meyerand ME (2001) Frequencies contributing to functional connectivity in the cerebral cortex in "resting-state" data. AJNR Am J Neuroradiol 22:1326-1333.

Cote KA, De Lugt DR, Campbell KB (2002) Changes in the scalp topography of event-related potentials and behavioral responses during the sleep onset period. Psychophysiology 39:29-37.

Diekelmann S, Born J (2010) The memory function of sleep. Nat Rev Neurosci 11:114-126.

Dimitriadis SI, Laskaris NA, Del Rio-Portilla Y, Koudounis GCh (2009) Characterizing dynamic functional connectivity across sleep stages from EEG. Brain Topogr 22:119-133.

Eguíluz VM, Chialvo DR, Cecchi GA, Baliki M, Apkarian AV (2005) Scalefree brain functional networks. Phys Rev Lett 94:018102.

Ferri R, Rundo F, Bruni O, Terzano MG, Stam CJ (2007) Small-world network organization of functional connectivity of EEG slow-wave activity during sleep. Clin Neurophysiol 118:449-456.

Ferri R, Rundo F, Bruni O, Terzano MG, Stam CJ (2008) The functional connectivity of different EEG bands moves towards small-world network organization during sleep. Clin Neurophysiol 119:2026-2036.

Fox MD, Raichle ME (2007) Spontaneous fluctuations in brain activity observed with functional magnetic resonance imaging. Nat Rev Neurosci $8: 700-711$

Hayasaka S, Laurienti PJ (2010) Comparison of characteristics between region-and voxel-based network analyses in resting-state fMRI data. Neuroimage 50:499-508.

He BJ, Raichle ME (2009) The fMRI signal, slow cortical potential and consciousness. Trends Cogn Sci 13:302-309.

He Y, Chen ZJ, Evans AC (2007) Small-world anatomical networks in the human brain revealed by cortical thickness from MRI. Cereb Cortex 17:2407-2419.

Horovitz SG, Fukunaga M, de Zwart JA, van Gelderen P, Fulton SC, Balkin TJ, Duyn JH (2008) Low frequency BOLD fluctuations during resting wakefulness and light sleep: a simultaneous EEG-fMRI study. Hum Brain Mapp 29:671-682.

Horovitz SG, Braun AR, Carr WS, Picchioni D, Balkin TJ, Fukunaga M, Duyn JH (2009) Decoupling of the brain's default mode network during deep sleep. Proc Natl Acad Sci U S A 106:11376-11381.

Johnson LC, Hanson K, Bickford RG (1976) Effect of flurazepam on sleep spindles and K-complexes. Electroencephalogr Clin Neurophysiol 40:67-77.

Kamada T, Kawai S (1989) An algorithm for drawing general undirected graphs. Inform Process Lett 31:7-15.

Kaufmann C, Wehrle R, Wetter TC, Holsboer F, Auer DP, Pollmächer T, Czisch M (2006) Brain activation and hypothalamic functional connectivity during human non-rapid eye movement sleep: an EEG/fMRI study. Brain 129:655-667.

Koch C (2009) The SCP is not specific enough to represent conscious content. Trends Cogn Sci 13:367.

Larson-Prior LJ, Zempel JM, Nolan TS, Prior FW, Snyder AZ, Raichle ME (2009) Cortical network functional connectivity in the descent to sleep. Proc Natl Acad Sci U S A 106:4489-4494.

Liu Y, Liang M, Zhou Y, He Y, Hao Y, Song M, Yu C, Liu H, Liu Z, Jiang T (2008) Disrupted small-world networks in schizophrenia. Brain 131:945-961.

Magnin M, Rey M, Bastuji H, Guillemant P, Mauguière F, Garcia-Larrea L (2010) Thalamic deactivation at sleep onset precedes that of the cerebral cortex in humans. Proc Natl Acad Sci U S A 107:3829-3833.

Maslov S, Sneppen K (2002) Specificity and stability in topology of protein networks. Science 296:910-913.

Massimini M, Ferrarelli F, Huber R, Esser SK, Singh H, Tononi G (2005) Breakdown of cortical effective connectivity during sleep. Science 309:2228-2232.

McCormick DA, Bal T (1997) Sleep and arousal: thalamocortical mechanisms. Annu Rev Neurosci 20:185-215.

Mesulam MM (2000) Principles of behavioral and cognitive neurology, Ed 2. Oxford: Oxford UP.

Meunier D, Achard S, Morcom A, Bullmore E (2009) Age-related changes in modular organization of human brain functional networks. Neuroimage 44:715-723.

Niiyama Y, Fujiwara R, Satoh N, Hishikawa Y (1994) Endogenous compo- nents of event-related potential appearing during NREM stage 1 and REM sleep in man. Int J Psychophysiol 17:165-174.

Ogilvie RD, Simons IA, Kuderian RH, MacDonald T, Rustenburg J (1991) Behavioral, event-related potential, and EEG/FFT changes at sleep onset. Psychophysiology 28:54-64.

Rechtschaffen A, Kales A, editors (1968) A manual of standardized terminology, techniques and scoring system of sleep stages in human subjects. Los Angeles: Brain Information Service/Brain Research Institute, University of California.

Rubinov M, Sporns O (2010) Complex network measures of brain connectivity: uses and interpretations. Neuroimage 52:1059-1069.

Salvador R, Suckling J, Schwarzbauer C, Bullmore E (2005) Undirected graphs of frequency-dependent functional connectivity in whole brain networks. Philos Trans R Soc Lond B Biol Sci 360:937-946.

Sämann PG, Wehrle R, Spoormaker VI, Hoehn D, Peters H, Holsboer F, Czisch M (2009) Development of the brain default mode network from wakefulness into slow wave sleep. In: Proceedings of the 17th Scientific Meeting of the International Society for Magnetic Resonance in Medicine. Honolulu.

Sporns O, Chialvo DR, Kaiser M, Hilgetag CC (2004) Organization, development and function of complex brain networks. Trends Cogn Sci $8: 418-425$

Stam CJ (2004) Functional connectivity patterns of human magnetoencephalographic recordings: a "small-world" network? Neurosci Lett 355:25-28.

Stam CJ (2010) Use of magnetoencephalography (MEG) to study functional brain networks in neurodegenerative disorders. J Neurol Sci 289:128-134

Stam CJ, Jones BF, Nolte G, Breakspear M, Scheltens P (2007) Small-world networks and functional connectivity in Alzheimer's disease. Cereb Cortex 17:92-99.

Stam CJ, de Haan W, Daffertshofer A, Jones BF, Manshanden I, van Cappellen van Walsum AM, Montez T, Verbunt JP, de Munck JC, van Dijk BW, Berendse HW, Scheltens P (2009) Graph theoretical analysis of magnetoencephalographic functional connectivity in Alzheimer's disease. Brain 132:213-224.

Steriade M, McCormick DA, Sejnowski TJ (1993) Thalamocortical oscillations in the sleeping and aroused brain. Science 262:679-685.

Steriade M, Contreras D, Amzica F (1994) Synchronized sleep oscillations and their paroxysmal developments. Trends Neurosci 17:199-208.

Supekar K, Musen M, Menon V (2009) Development of large-scale functional brain networks in children. PLoS Biol 7:e1000157.

Tononi G (2004) An information integration theory of consciousness. BMC Neurosci 5:42.

Tononi G, Massimini M (2008) Why does consciousness fade in early sleep? Ann N Y Acad Sci 1129:330-334.

Tononi G, Sporns O (2003) Measuring information integration. BMC Neurosci 4:31.

Tzourio-Mazoyer N, Landeau B, Papathanassiou D, Crivello F, Etard O, Delcroix N, Mazoyer B, Joliot M (2002) Automated anatomical labeling of activations in SPM using a macroscopic anatomical parcellation of the MNI MRI single-subject brain. Neuroimage 15:273-289.

van den Heuvel MP, Stam CJ, Boersma M, Hulshoff Pol HE (2008) Smallworld and scale-free organization of voxel-based resting-state functional connectivity in the human brain. Neuroimage 43:528-539.

van den Heuvel MP, Stam CJ, Kahn RS, Hulshoff Pol HE (2009) Efficiency of functional brain networks and intellectual performance. J Neurosci 29:7619-7624

Wang J, Wang L, Zang Y, Yang H, Tang H, Gong Q, Chen Z, Zhu C, He Y (2009) Parcellation-dependent small-world brain functional networks: a resting-state fMRI study. Hum Brain Mapp 30:1511-1523.

Watts DJ, Strogatz SH (1998) Collective dynamics of 'small-world' networks. Nature 393:440-442.

Weissenbacher A, Kasess C, Gerstl F, Lanzenberger R, Moser E, Windischberger C (2009) Correlations and anticorrelations in resting-state functional connectivity MRI: a quantitative comparison of preprocessing strategies. Neuroimage 47:1408-1416.

Zhang D, Snyder AZ, Fox MD, Sansbury MW, Shimony JS, Raichle ME (2008) Intrinsic functional relations between human cerebral cortex and thalamus. J Neurophysiol 100:1740-1748. 